\title{
Human Factors Approach in the Design of an Electronic Medication Management System for Preventing Inpatient Medication Errors
}

Elisabetta Volpi ${ }^{1}$, Alessandro Giannelli ${ }^{2}$, Giulio Toccafondi ${ }^{3 *}$, Riccardo Tartaglia ${ }^{3}$, Mauro Micalizzi ${ }^{4}$, Maurizio Mangione ${ }^{4}$, Monica Baroni ${ }^{5}$, Sara Tonazzini ${ }^{1}$, Stefania Alduini ${ }^{1}$, Stefania Biagini ${ }^{1}$, Elaine Laws $^{6}$ and Tommaso Bellandi ${ }^{3}$

${ }^{1}$ Hospital Pharmacy, Fondazione Toscana G. Monasterio, Heart Hospital, Massa, Italy

${ }^{2}$ Hospital Pharmacy student, University of Pisa, Italy

${ }^{3}$ Clinical Risk Management and Patient Safety Center, Tuscany Region, Florence, Italy

${ }^{4}$ Department of Informatics, Fondazione Toscana G. Monasterio, Italy

${ }^{5}$ Clinical Risk Manager, Fondazione Toscana G. Monasterio, Italy

${ }^{6}$ Nursing Administration, Fondazione Toscana G. Monasterio, Heart Hospital, Massa, Italy

\begin{abstract}
Background: In order to ensure quality and safety of prescriptions, electronic medication management system needs to comply with medication safety requirements and human factors principles. The design of such a system can make the difference on physician acceptance and consequently on medication safety. The aim of the study is to evaluate to what extent the changes in the design of the electronic medication management system may affect the
\end{abstract} rate of medication errors.

Materials and method: In light of the growing development of computerized systems in health services it has become necessary to design electronic medication management system for drug prescription and administration compliant with the requirements of medication safety practices.

Prescription data relative to 100 patients admitted to the Cardiothoracic Department of the Gabriele Monasterio Foundation Heart Hospital (FTGM) were extracted from the computerized medical notes from March 2013 until May 2013. The prescriptions examined had been written using the electronic documentation prompt without structured entry fields. All prescriptions were evaluated using the medication safety requirements of the medication safety practice developed by the Centre for Patient Safety of the Regional Department of Health in Tuscany, Italy. The same prescriptions were then simulated using the novel electronic medication management module and reassessed according to the safety requirements.

Results: Of the 4112 prescriptions pertaining to the 100 study patients analyzed, $88.5 \%$ were found to be erroneous or incomplete. In particular $46.8 \%$ did not include the route of administration, $29.4 \%$ the pharmaceutical form, $10.6 \%$ the number of administrations per day and/or the time of administration, in $8.2 \%$ the dose was not defined and $4.9 \%$ did not include the active pharmaceutical agent or trade name. $14.9 \%$ were considered with high potential for harm. The same prescriptions simulated through the novel electronic module were $99.1 \%$ correct and complete.

Conclusions: The ergonomic design of the module for electronic prescription meets the medication safety requirements and has a role in reducing drug errors and enhancing the safety of the workflow. The module introduced structured fields pertaining the type of drug being prescribed which were positively embodied in the routine and produced a significant reduction of prescription errors.

Keywords: Medication safety; Human factors; Design; Computerized medication management

\section{Introduction}

Every patient has the $5.5 \%$ probability to suffer the consequences of a medication error during an average hospital stay. The risk increases by $0.5 \%$ for every additional night spent in the hospital [1]. Medication errors have important implications for patient safety (e.g., increased length of hospitalization, drug-related adverse events, and increased mortality) and their identification is a main target in improving clinical practice. Hospital electronic prescribing system offers to healthcare professionals a powerful tool for safely and efficiently managing patients' medications. The use of structured software application has been shown to improve the quality of the information on patient treatment during hospitalization by reducing incomplete and inappropriate prescriptions and medication errors [2]. Nevertheless the impact of an electronic medication management system on safety depends on how the design fits the specific context and how the workflow of healthcare workers is affected [3]. The way in which the documentation flow is made effective by electronic medical records dramatically changes the proportion of time spent on patient care rather than on documenting care. Early implementation of CPOE may produce unexpected systemic drawbacks which may be attributable to workflow disruptions such as excessive steps to obtain usual medications or persistent high rates of ADEs after the implementation of CPOE [4,5]. EMR and CPOE modules enhance the individual capability of accessing medical information. Such digital technologies fully unfolds their potential when seamlessly support the interactions

*Corresponding author: Giulio Toccafondi, Clinical Risk Management and Patient Safety Center, Tuscany Region, Florence, Italy, Tel: +390557949615; E-mail: toccaf@gmail.com

Received July 17, 2015; Accepted August 05, 2015; Published August 11, 2015

Citation: Volpi E, Giannelli A, Toccafondi G, Tartaglia R, Micalizzi M, et al. (2015) Human Factors Approach in the Design of an Electronic Medication Management System for Preventing Inpatient Medication Errors. J Pharmacovigilance S2: 006. doi:10.4172/2329-6887.S2-006

Copyright: @ 2015 Volpi E, et al. This is an open-access article distributed under the terms of the Creative Commons Attribution License, which permits unrestricted use, distribution, and reproduction in any medium, provided the original author and source are credited. 
between the health care workers and the different components of health care through transparent and structured workflow [6], including the key tasks of prescription and administration within the medication process at in-hospital settings.

The American Institute of Medicine estimated that, on average, hospitalized patients are subject to at least one medication error per day [7]. They also recommend the use of electronic prescribing through a computerized provider order entry system to reduce medication errors and patient harm [8].

Since 1995, it was observed in a prospective cohort study that around $6,5 \%$ of patients admitted in hospital underwent serious adverse drug events related to medication errors, of which $1 \%$ resulted in patient death, $12 \%$ was life-threatening and 30\% caused a serious drug reaction [9]. In this study it was also observed that the main causes for the medication errors were at the stages of ordering (56\%) and administration (34\%); transcription (6\%) and dispensing errors (4\%) were less common. CPOE have been regarded as tool to improve the quality and safety of healthcare workers, increasing time efficiency and compliance with medication safety requirements [10]. Nevertheless, CPOE need to be efficiently designed for supporting the process of entering and retrieving information and the communication and coordination activities that the CPOE is meant to support. In this regard the digital technologies needs to proficiently couple with the pre-existing social structure and the given organizational matrix in order to avoid mismatch and latent failures [11].

Since 2009, the Centre for Patient Safety of the Regional Department of Health in Tuscany, Italy, promoted a campaign for the adoption of the "five rights" [12] of medication safety for a correct and complete prescription and administration, in order to minimize the exposure to medication errors. Efforts for ensuring each of requirements for medication safety must take into account human factor and ergonomic design issues.

In the regional Tuscan health care system, a recent investigation identified the lack of an electronic health records, including an electronic prescribing system, as one of the factors influencing medication errors at hospital.

Therefore, an in-house electronic prescribing software module was designed within the electronic health record system already in use at the Cardiothoracic Department of the Gabriele Monasterio Foundation Heart Hospital (FTGM). A human factors approach was used in order to identify sound design patterns eligible for standardized requirements. The health care workers were involved into the design of their own medication management system.

The module offers drug selection and dosing. It supports the drug administration workflow providing access to pharmacy and nurse for a comprehensive integration of the involved stakeholders into the medication processes. The aim was to minimize medication errors, drug-related adverse events and improve patient safety. The module does not feature decision support algorithms.

According to the human factors design approach, a safe prescribing interface should minimize human memory load and promote recognition rather than recall [13]. The system generates prescriptions that are clear and complete in all their formal aspects through a structured and codified order data entry, and it communicate directly all prescriptions to scheduling software for administration, so it would reduce the risk of handwriting or verbal misinterpretation, erroneous transcriptions or inaccurate communication.
The medication management system provides the clinicians with direct links to the information required for effectively choosing the type of drug, the dose and correct compilation of all the fields required for a safe administration.

The aim of the present study was to test to what extent the new electronic prescribing system module of the EMR reduces opportunities for errors in the medication prescription process and bridges gaps in care flows (i.e., lack of information, misinterpretations) which may cause hazard to patient safety. For assessing the safety of the novel electronic prescribing module, we compared the same set of 4112 prescriptions with the pre-existing electronic documentation prompts of the EMR and then simulated through the electronic medication management system, designed according to human factors principles.

\section{Materials and Methods}

We retrospectively enrolled 100 consecutive patients admitted to the Cardiothoracic Department of the FTGM Heart Hospital from March 2013 until May 2013.

We extracted their prescription data entered during hospitalization. The prescription had been written using the traditional method of free text, from the computerized medical notes. All prescriptions were analyzed to evaluate errors and the same prescriptions were then simulated using the electronic prescription program to compare the two digital technologies and to see if the electronic system reduced errors.

Two hospital pharmacists, the clinical risk manager, who is a cardiologist and a nurse, evaluated the prescriptions. A correct and complete prescription needs to fulfill the following elements: a) name of the commercial drug or active molecule; b) dose, including measurement unit (i.e., $\mathrm{mg}, \mathrm{ml}, \mathrm{UI}$ ); $\mathrm{c}$ ) if it is an IV drug the indication of the speed of administration, expressed as weight/time or volume/time is needed; d) pharmaceutical formulation; e) route of administration; f) medication administration timing; g) duration of prescriptions; h) name of the prescribing physician.

As a consequence, for the evaluation of medication errors we indicated six different types of error. In particular we considered as incomplete prescription when 1) the name of the commercial drug or active molecule was missing; 2 ) the dose was missing or incomplete (e.g., dose without measurement units); 3) the pharmaceutical formulation was missing; 4) route of administration was missing; 5) medication administration timing was missing, 6) speed of administration, in case of IV drugs was missing. The use of abbreviations that are ambiguous, or that in their written form might be confused with something else, was considered as medication errors.

In order to test the reliability of the electronic prescription program, we evaluated the number of medication errors generated using the two different digital technologies of prescribing using the same criteria.

\section{Results}

We analyzed 4112 prescriptions pertaining to the 100 study patients analyzed. Patient were equally distributed among those underwent cardiac invasive procedures or pharmacological treatments (43\%) and those underwent cardiac surgery (57\%). The mean duration of hospitalization was 4,6 days. We observed that the mean number of prescriptions/patient was 27.4 during hospitalization, corresponding to 5.9 prescriptions / day for each patient.

We found that $88.5 \%$ of prescriptions $(n=3641)$ were erroneous or incomplete. The total number of errors observed was $n=5134$ and we 
found frequently more than one error/prescription (i.e., prescription with missing route of administration and pharmaceutical formulation). The type of errors was various; in particular we found $4.9 \%(\mathrm{n}=$ 253) missing name of the commercial drug or active molecule, $8.2 \%$ $(\mathrm{n}=422)$ missing dose, $29.4 \%(\mathrm{n}=1509)$ missing pharmaceutical formulation, $46.8 \%(\mathrm{n}=2404)$ missing route of administration, $10.6 \%$ $(\mathrm{n}=546)$ missing medication administration timing schedule.

We also observed 69\% $(\mathrm{n}=175)$ prescriptions with missing name of active molecule or brand name, IV drugs without the definition of the diluent needed for the preparation of the IV solution, $26 \%(n=66)$ of prescriptions made with unofficial or ambiguous abbreviations and $5 \%(n=12)$ of typing errors that made the prescription unclear.

Among prescriptions that presented lack of definition of the dose, $63.0 \%(n=266)$ were IV drugs without definition of the volume of the diluent, $28.9 \%(\mathrm{n}=122)$ did not indicate the dose of the drug prescribed and $8.1 \%(n=34)$ were prescriptions of oral anticoagulants without definition of the dose nor notes about the wait for INR results from the laboratory. $5.4 \%(\mathrm{n}=81)$ of prescriptions without information about the pharmaceutical formulation would have been at risk of wrong interpretation due to the presence of drugs with the same active molecule but different formulations. Among prescriptions that presented lack of definition of the route of administration, $4.4 \%$ $(\mathrm{n}=106)$ were represented by prescriptions that may induce incorrect administration by the nursing staff due to the availability of different formulations that can be administered using different routes. Finally, among prescriptions without details about the administration timing schedule we found $6.6 \%$ of prescription at risk of medication errors caused by the lettering "as needed" without additional indications about the clinical condition (e.g., lab parameters, pain assessment score) which authorize their administration or information about the maximum single dose and the interval between doses.

Reviewing all incomplete prescriptions $(\mathrm{n}=3641)$ we noted that of the overall percentage of erroneous or incomplete prescriptions $14.9 \%(\mathrm{n}=543)$ were at high risk of misinterpretation by the nursing staff and consequently for administration errors. For example, the IV prescriptions without a clear identification of the diluent or the prescriptions with the lettering "as needed", as above mentioned.

The analysis of the same prescriptions $(n=4112)$ simulated using the new electronic prescribing system showed that $99.1 \%$ of them $(\mathrm{n}=$ $4076)$ were correct and complete. The incomplete prescriptions $(0.9 \%$, $\mathrm{n}=36$ ) were represented by drug orders in which it was reported an "as needed" note without a complete description of the clinical conditions that allow the nursing staff to administer the drug. Even if the maximum single dose and the interval between doses were well defined using the electronic prescribing system, these prescriptions still need to be considered incomplete because they leave room for incorrect interpretation, as previously described.

\section{Discussion and Conclusions}

Adverse events related to medication errors represent the main cause of patient injury during hospitalization and they can occur throughout the overall in-hospital medication process.

The prescription and administration moments have a direct effect on patient health; therefore all healthcare settings should improve the safety of their prescribing system.

We demonstrated that, after an ergonomic redesign, our electronic prescribing system is fully compliant with the requirements of the
Patient Safety Practice for medication safety adopted by the providers of the regional health care system.

In fact we observed a drastic reduction of the incomplete or incorrect prescriptions from $88.5 \%$ to $0.9 \%$, identified when using the traditional method of free text.

The new system completely eliminated all ambiguous abbreviations and the drug orders in which the physician did not indicate the route of administration, the pharmaceutical formulation or the medication administration timing schedule. The residual proportion of incomplete prescriptions due to the "as needed" note without additional details regarding the indications about the clinical condition (e.g., lab parameters, pain score) that authorize the administration, needs to be solved with an in-hospital procedure, in collaboration with the Clinical Risk manager, who regulate such circumstances.

In particular we noticed that all missing information that we found with the free text method (e.g., dose, route of administration, pharmaceutical formulation, time schedule) have been eliminated using the new system. When a physician prescribes a medication using the electronic prescribing system all items must be specified so that the prescription doctor can be validated. This explains why the use of CPOE provides $100 \%$ of prescriptions that fulfill all required elements. This represents a remarkable achievement of CPOE in order to minimize the risk of medication errors.

It was observed [14] that by using $\mathrm{CPOE}$ clinicians obtained the complete elimination of prescriptions in which the route of administration or dosage were missing.

Similarly [15] a CPOE was able to reduce the amount of incomplete prescriptions due to lack administration route definition or dosage definition.

The only prescriptions that have not been correctly defined using our electronic prescribing system is the use of lettering "as needed", without additional indications about the clinical condition (e.g., lab parameters, pain assessment score) that authorize the nurse to administer the medication. In order to define this situation, we are preparing, a "see and treat" protocol, with the list of drugs and the clinical condition or lab parameters that allow the administration by the nursing staff, in collaboration with the Centre for Patient Safety and our medical staff.

So, CPOE has demonstrated to have a substantial potential for improving the quality and safety of prescriptions, avoiding lack of information and increasing time efficiency and compliance with medication safety requirements. It may need to be integrated with local protocols for the use of particular class of drugs, in order to minimize the risk of incorrect interpretation.

Besides, the ergonomic design of the new system proved to be effective in guiding and supporting the physician at the stage of prescription. The simulation of the prescription is a limitation of the study that cannot demonstrate the impact of the solution on the real practice involving also the pharmacists and the nurses. The context played a significant role to facilitate the innovation and the constructive collaboration between clinicians, computer scientists and ergonomists: the structure is a high level specialist hospital with an embedded research unit, plus an experience of a decade at the in-house design and management of an electronic patient record. These characteristics must be taken into account for the potential transferability of this intervention. 
Citation: Volpi E, Giannelli A, Toccafondi G, Tartaglia R, Micalizzi M, et al. (2015) Human Factors Approach in the Design of an Electronic Medication Management System for Preventing Inpatient Medication Errors. J Pharmacovigilance S2: 006. doi:10.4172/2329-6887.S2-006

Electronic medications management is an important part of the medical services offered to the patient. The presence of all requirements that identify a correct and complete prescription is a fundamental aspect in order to assure the efficacy of the pharmacological treatment, enhance patient's compliance and reduce the risk of drug related adverse events. Future features regarding the prescribing and administration actions should be considered in order to improve patient safety as the supplement of alerts for drug-drug interactions or high-risk drugs (e.g., oral warfarin, IV potassium chloride) and the barcode reader software for medications check and patient identification. Moreover, medications reconciliation at the time of admission and after discharge is an important topic for patient safety that was not taken into account in this applied research. The lack of integration between hospital records and the long patient history in charge of the general practitioner is to date the main barrier that needs to be targeted in our healthcare system in order to coordinate the efforts for medication safety.

\section{Acknowledgement}

We thank our colleagues of the Regional Clinical Risk Management and Patient safety Center (Italy) for assistance with the evaluation of the ergonomic aspects of the software.

\section{References}

1. Hauck K, Zhao X (2011) How dangerous is a day in hospital? A model of adverse events and length of stay for medical inpatients. Medical Care 49: 1068-1075.

2. Kaushal R, Shojania KG, Bates DW (2003) Effects of computerized physician order entry and clinical decision support systems on medication safety: A systematic review. Arch Intern Med 163: 1409-1416.

3. Carayon P, Wetterneck TB, Alyousef B, Brown RL, Cartmill RS, et al. (2015) Impact of Electronic Health Record technology on the work and workflow of physicians in the intensive care unit. Int J Med Inform 84: 578-594.
4. Nebeker J, Hoffman J, Weir CR (2005) High rates of adverse drug events in a highly computerized hospital. Arch Intern Med 165: 1111-1116.

5. Holden RJ (2010) Physicians' beliefs about using EMR and CPOE: In pursuit of a contextualized understanding of health It use behavior. Int J Med Inform 79: $71-80$.

6. Ash JS, Berg M, Coiera E (2004) Some unintended consequences of information technology in health care: The nature of patient care information system-related errors. J Am Med Inform Assoc 11: 104-112.

7. Aspden P, Wolcott J, Bootman J, Cronenwett LR (2007) Preventing medication errors. The National Academies Press, Washington DC.

8. Committee on Quality of Health Care in America (2001) Using information technology in crossing the quality chasm: A new health system for the $21 \mathrm{st}$ century. Institute of Medicine, National Academy Press, Washington DC.

9. Bates DW, Cullen DJ, Laird N, Petersen LA, Small SD, et al. (1995) Incidence of adverse drug events and potential adverse drug events. Implications for prevention. ADE Prevention Study Group. JAMA 274: 29-34.

10. Campanella P, Lovato E, Marone C, Fallacara L, Mancuso A, et al. (2015) The impact of electronic health records on healthcare quality: A systematic review and meta-analysis. Eur J Public Health.

11. Reason J (1995) Understanding adverse events: Human factors. Quality in Health Care 4: 80-89

12. Grissinger MA (2010) The Five Rights Destination Without a Map. PT 35: 542.

13. Zhang J, Johnson TR, Patel VL, Paige DL, Kubose T (2003) Using usability heuristics to evaluate patient safety of medical devices. Journal of Biomedical Informatics 36: 23-30.

14. Evans KD, Benham SW, Garrard CS (1998) A comparison of handwritten and computer-assisted prescriptions in an intensive care unit. Crit Care 2: 73-78.

15. Westbrook JI, Reckmann M, Li L, Runciman WB, Burke R, et al. (2012) Effects of two commercial electronic prescribing systems on prescribing error rates in hospital in-patients: a before and after study. PLoS Med 9: e1001164
This article was originally published in a special issue, Medication Errors handled by Editor(s). Dr. Prashansa Agrwal, University of Arizona, USA 